\title{
ESTUDO DE CARACTERIZAÇÃO DO FRUTO CAMBUCI [Campomanesia phaea (O. Berg.) Landrum] E SUA APLICAÇÃO NO PROCESSAMENTO DE GELEIA
}

\author{
IGOR GALVÃO SILVA * \\ ANGELA DE FATIMA KANESAKI CORREIA** \\ JOSEANE THEREZA BIGARAN*** \\ CAMILA PADOVAN BAPTISTA**** \\ LEANDRO FRANCISCO DO CARMO***** \\ MARTA HELENA FILLET SPOTO******
}

\begin{abstract}
O objetivo deste trabalho foi estudar possível diferença de variedades de cambuci, informada por produtores, mediante a caracterização do fruto e a elaboração de geleia. Os frutos foram separados pelas quatro possíveis variedades, denominadas $A, B$, $\mathrm{C}$ e $\mathrm{D}$, e analisados quanto ao peso médio, cinzas, umidade, $\mathrm{pH}$, sólidos solúveis, acidez titulável, ratio, ácido ascórbico e atividade de água. Para a elaboração da geleia foi escolhida a variedade A com duas formulações, $50 \% 50 \%$ e $40 \% 60 \%$ fruto e açúcar, respectivamente. Para as possíveis variedades de cambuci, somente a atividade de água não apresentou diferença significativa. A acidez e o ratio da variedade $B$ chamam a atenção, pois diferiram das demais indicando fruto mais propício ao consumo in natura. Em todas as outras análises existiu diferença estatística de pelo menos uma variedade, mas esses dados devem ser vistos com cautela por sofrerem influência de condições edafoclimáticas. A amostra de geleia com $60 \%$ de açúcar apresentou resultados menores para $\mathrm{pH}$, acidez, luminosidade e ${ }^{\circ}$ hue, o que contribuiu para os melhores resultados no teste de preferência em todos os atributos avaliados (aparência, cor, sabor e textura), exceto quanto ao aroma em que ambas as amostras (50\% e 60\%) não diferiram.
\end{abstract}

PALAVRAS-CHAVE: CAMBUCIZEIRO; MIRTÁCEAS; GELEIA; ANÁLISE SENSORIAL.

* Mestrando em Energia Nuclear na Agricultura e no Ambiente, Centro de Energia Nuclear na Agricultura (CENA), Universidade de São Paulo (USP), Piracicaba, SP (e-mail: galvaoigor@yahoo.com.br).

** Doutoranda em Ciência e Tecnologia de Alimentos, Escola Superior de Agricultura "Luiz de Queiroz", USP, Piracicaba, SP (e-mail: afkcorreia@gmail.com).

*** Mestranda em Ciência e Tecnologia de Alimentos, Escola Superior de Agricultura "Luiz de Queiroz", USP, Piracicaba, SP (e-mail: jobigaran@hotmail.com).

**** Graduanda em Engenharia de Alimentos, Universidade Metodista de Piracicaba (UNIMEP), Santa Bárbara d'Oeste, SP (e-mail: cabaptista2011@gmail.com).

***** Pós-Doutor em Recursos Florestais não Madereiros, Escola Superior de Agricultura "Luiz de Queiroz", USP, Piracicaba, SP (e-mail: drleandrocarmo@gmail.com).

****** Pós-doutora pela Universidade de São Paulo, professora, Escola Superior de Agricultura "Luiz de Queiroz", USP, Piracicaba, SP (e-mail: mhfspoto@esalq.usp.br). 


\section{INTRODUÇÃo}

O cambuci ou cambucizeiro [Campomanesia phaea (O. Berg.) Landrum], da família das Mirtáceas, é uma fruta originária do Brasil. Trata-se de espécie frutífera silvestre nativa da serra do Mar e Mata Atlântica, entre os estados de São Paulo e Minas Gerais. É também encontrada em outras regiões do Brasil, mas em menor proporção (MALUF e EREIO, 2005). O fruto, conhecido desde os primórdios coloniais, foi usado durante muitos anos exclusivamente como aromatizante para cachaças (MATHIAS e ANDRADE, 2011). A espécie (Campomanesia phaea) foi inicialmente descrita como Abbevillea phaea por Berg, em 1857. Grande parte das plantações de cambuci está localizada nas cidades de Rio Grande da Serra, Santo André (na Vila de Paranapiacaba), Salesópolis, Biritiba-Mirim, Paraibuna e outras cidades com resquícios de Mata Atlântica (SLOW FOOD BRASIL, 2011). A espécie está ameaçada de extinção devido à exploração predatória da sua madeira para fabricação de ferramentas e também pelo desmatamento da Mata Atlântica (KAWASAKI e LANDRUM, 1997).

As características do fruto incluem casca fina e coloração verde, inclusive com a maturidade (tornando-se às vezes um pouco mais amarelada). Desse modo, o fruto amadurece quando fica mais macio e cai dos galhos. Apresenta cheiro doce, mas é extremamente ácido como o limão (KAWASAKI e LANDRUM, 1997). Apesar de rico sob os pontos de vista nutricional e sensorial, o consumo da fruta in natura é limitado devido à forte acidez. Como as qualidades de sabor e aroma não se perdem com o processamento, o cambuci é bastante utilizado como aromatizante e no preparo de sucos, geleias e doces em calda (MATHIAS e ANDRADE, 2011).

No Brasil existem grandes problemas de desperdício e aproveitamento de frutos impróprios para a comercialização. Nessa esteira surge como alternativa a elaboração de geleia, que se mostra valiosa opção por ter boa aceitação sensorial e não exigir o mesmo padrão de qualidade imposto para comercialização do fruto. A adição de açúcar, junto com a concentração a elevadas temperaturas no processamento da geleia, promovem considerável estabilidade microbiológica, reduzindo a carga microbiana e a atividade de água do produto. Assim, os produtores encontram nas indústrias processadoras a garantia para a comercialização do fruto. Já as indústrias, podem utilizar matéria-prima mais barata e agregar valor ao fruto (BELTRÃO FILHO e DA SILVA, 2006; KAWASAKI e LANDRUM, 1997).

O fruto ameaçado de extinção carece de maiores estudos, pois os produtores relatam diferenciação de espécies não encontrada na literatura com relação ao seu tamanho, formato, coloração, acidez e sabor. Nesse contexto, o objetivo deste trabalho foi estudar possível diferença de variedades de cambuci, informada por produtores, mediante a caracterização do fruto e a elaboração de geleia.

\section{MATERIAL E MÉTODOS}

Os frutos analisados foram coletados na região de Mogi Mirim, interior do estado de São Paulo, separados pelas quatro possíveis variedades, denominadas $A, B, C$ e $D$, acondicionados em embalagem de polietileno e encaminhados ao Laboratório de Frutas e Hortaliças, da Escola Superior de Agricultura Luiz de Queiroz (ESALQ/USP), sendo armazenados em temperatura de congelamento $\left(-18^{\circ} \mathrm{C}\right)$ para posterior análise.

Para realizar a caracterização do fruto, as amostras foram processadas com mixer vertical Black e Decker (SB40) e pesadas para as análises. Determinou-se a umidade segundo o método $\mathrm{n}$. 950.46 da AOAC e o pH pelo método n. 981.12 (AOAC, 2005), utilizando-se potenciômetro (TEC3$\mathrm{MP}$, Tecnal). A acidez titulável, expressa em g ácido cítrico $100 \mathrm{~g}^{-1}$ polpa, foi determinada pelo método 942.15 da AOAC e o conteúdo de sólidos solúveis totais ( $\left.{ }^{\circ} B r i x\right)$ quantificado em refratômetro Auto Abbe, modelo 10500/10501, Lidaa (com correção de temperatura), segundo o método n. 932.12 da AOAC (2005). A atividade de água (aw) foi determinada de forma direta em medidor de atividade de 
água, Testo 650 , da Instrucamp e o ratio pela razão sólidos solúveis totais e acidez titulável.

O conteúdo de ácido ascórbico das amostras foi quantificado por titulação com o indicador DCFI (2,6-diclorofenolindofenol-sódio) de acordo com a metodologia proposta por Strohecker e Henning (1967) e a cor por reflectometria. Utilizou-se colorímetro Color Meter-Minolta 200b, com leitura direta de valores de $L^{*}$ (luminosidade), ${ }^{\circ}$ hue (matiz) e croma (saturação), conforme sistema proposto pela Comission Internacionale de L'Eclaraige (CIE) (KONICA MINOLTA, 1998).

Escolheu-se para a elaboração das geleias o cambuci A, sendo os frutos selecionados, lavados em água corrente e em seguida imersos em solução de cloro a $200 \mathrm{mg} \mathrm{L}^{-1}$ (Dicloro Isocianurato de Sódio Dihidratado - 3\% de cloro ativo), durante 15 minutos e enxaguados em água corrente tratada. Em seguida, os frutos foram processados inteiros com o auxílio de mixer vertical Black e Decker (SB40), pesados e misturados com açúcar na proporção de 50\%50\% e 40\%60\% fruto e açúcar, respectivamente. A mistura, previamente uniformizada até obtenção de massa homogênea, foi submetida ao processo de aquecimento e concentração. Devido à acidez elevada do fruto não foi adicionado ácido cítrico na elaboração da geleia, mas acrescentou-se aproximadamente $2 \%$ de pectina cítrica (Vertec) quando o produto atingiu concentração de $65^{\circ}$ Brix. Isso porque a adição de pectina no início do processo poderia levar ao rompimento das cadeias pécticas pela acidez e principalmente pelo binômio tempo $x$ temperatura (MEDINA, 1985). As geleias foram processadas em recipientes de aço inoxidável com sistema de aquecimento até a concentração final de sólidos solúveis de $69^{\circ} \mathrm{Brix}$, embaladas à quente em recipientes de vidro e armazenadas em temperatura de refrigeração $\left(8^{\circ} \mathrm{C}\right)$.

A análise sensorial ocorreu em cabines individuais do Laboratório de Análise Sensorial do Departamento de Agroindústria Alimentos e Nutrição, ESALQ/USP, sendo as amostras avaliadas pelo método afetivo por 52 julgadores não treinados. As amostras de geleia de cambuci, codificadas com números aleatórios de três dígitos, foram servidas em elemento neutro, dispersas em torrada, sendo apresentadas de forma monádica e balanceada para minimizar o efeito da ordem de apresentação.

Foram aplicados testes de aceitação, utilizando-se escala hedônica estruturada de sete pontos, variando entre os termos "desgostei muito (escore 1)" e "gostei muito (escore 7)" para os atributos de aparência, cor, sabor e textura da geleia, bem como o teste de preferência pareado monocaudal de acordo com Meilgaard, Civille e Carr (1991).

Os resultados para caracterização dos frutos e da análise sensorial foram expressos em médias e desvio padrão, sendo submetidos à análise de variância e teste de comparação múltipla de Tukey, ao nível a de probabilidade de $5 \%(p<0,05)$, utilizando-se o Software Action versão 1.1/ 2.5.1 (PORTAL ACTION, 2011). Todas as análises para caracterização dos frutos foram realizadas em triplicata.

\section{RESULTADOS E DISCUSSÃO}

$\mathrm{Na}$ Tabela 1 são apresentados os resultados analíticos obtidos na caracterização das possíveis variedades $(A, B, C$ e $D)$ de cambuci.

Em relação ao peso médio, as variedades $A, C$ e $D$ não diferiram estatisticamente. $A$ variedade $B$ apresentou o maior valor, pesando 47,56 gramas e diferindo de $A$ e $C$, mas não da amostra $D$ (com $34,32 \mathrm{~g}$ ). A variedade $A$ não diferiu de $B$ e $C$ quanto ao teor de cinzas ou sais minerais totais e os valores para cinzas de $C$ e $D$ não diferiram estatisticamente. As variedades apresentaram valor médio de $2,71 \%$ de sais minerais, podendo ser comparada à média $(2,50 \%)$ das amêndoas da castanha de cajú (LIMA, GARCIA e LIMA, 2004) e de castanha-do-Brasil (3,84\%) (SOUSA e MENEZES 2004) (Tabela 1). De acordo com Franco (1999), os sais minerais (como cálcio, fósforo, sódio, potássio e magnésio, dentre outros) estabelecem o equilíbrio físico-químico do organismo, regulando o ritmo cardíaco, a respiração e a digestão. 
TABELA 1 - CARACTERIZAÇÃO DAS QUATRO PROVÁVEIS VARIEDADES DO CAMBUCI

\begin{tabular}{ccccc}
\hline & \multicolumn{4}{c}{ Variedades } \\
\cline { 2 - 5 } Análises & A & B & C & D \\
\cline { 2 - 5 } & $22,84^{\mathrm{a}} \pm 5,85$ & $47,56^{\mathrm{b}} \pm 2,59$ & $24,00^{\mathrm{a}} \pm 0,35$ & $34,32^{\mathrm{ab}} \pm 15,95$ \\
Peso médio (g) & $2,66^{\mathrm{ab}} \pm 0,12$ & $2,48^{\mathrm{a}} \pm 0,05$ & $2,77^{\mathrm{bc}} \pm 0,03$ & $2,94^{\mathrm{c}} \pm 0,15$ \\
Cinzas (\%) & $86,33^{\mathrm{a}} \pm 0,14$ & $87,14^{\mathrm{a}} \pm 0,07$ & $89,79^{\mathrm{b}} \pm 0,05$ & $88,20^{\mathrm{ab}} \pm 1,46$ \\
Umidade (\%) & $2,97^{\mathrm{a}} \pm 0,02$ & $2,95^{\mathrm{a}} \pm 0,01$ & $2,78^{\mathrm{b}} \pm 0,01$ & $2,75^{\mathrm{b}} \pm 0,01$ \\
pH & $13,06^{\mathrm{a}} \pm 0,08$ & $12,90^{\mathrm{a}} \pm 0,03$ & $8,68^{\mathrm{b}} \pm 0,01$ & $11,44^{\mathrm{c}} \pm 0,15$ \\
Sólidos solúveis ( ${ }^{\circ}$ Brix) & & & \\
Acidez titulável (g ácido & $5,32^{\mathrm{a}} \pm 0,32$ & $2,55^{\mathrm{b}} \pm 0,09$ & $4,91^{\mathrm{a}} \pm 0,06$ & $5,76^{\mathrm{c}} \pm 0,08$ \\
cítrico 100 g-1) & $2,45^{\mathrm{a}} \pm 0,15$ & $5,05^{\mathrm{b}} \pm 0,18$ & $1,77^{\mathrm{c}} \pm 0,03$ & $1,99^{\mathrm{c}} \pm 0,05$ \\
Ratio & & & & \\
Ácido ascórbico (mg & & & \\
100 g-1) & $35,44^{\mathrm{a}} \pm 0,64$ & $16,79^{\mathrm{b}} \pm 1,93$ & $13,05^{\mathrm{c}} \pm 0,64$ & $47,38^{\mathrm{d}} \pm 1,29$ \\
Atividade de água (aw) & $0,97^{\mathrm{a}} \pm 0,00$ & $0,98^{\mathrm{a}} \pm 0,00$ & $0,98^{\mathrm{a}} \pm 0,01$ & $0,97^{\mathrm{a}} \pm 0,01$ \\
\hline
\end{tabular}

Diferentes letras minúsculas na mesma linha indicam diferença estatística $(p<0,05)$ entre as possíveis cultivares de cambuci.

Os resultados obtidos para umidade das variedades $A, B$ e $D$ do cambuci não diferiram estatisticamente, tendo a variedade $C$ apresentado o maior valor $(89,79 \%)$ e não diferindo da variedade D. Os resultados obtidos ficaram próximos aos encontrados por Vallilo et al. (2005) para cambuci (88\%), cuja elevada concentração de água é característica dos frutos carnosos e suculentos da família Myrtaceae (LEGRAND e KLEIN, 1977). Para o pH ocorreu variação de 2,97 a 2,75, não sendo verificadas diferenças estatísticas entre as variedades $A$ e $B$ e as variedades $C$ e $D$ (Tabela 1). Tais valores são coerentes com o estudo realizado por Vallilo et al. (2005), que encontraram pH 2,91 para o fruto do cambuci.

O teor de sólidos solúveis expresso em ${ }^{\circ}$ Brix depende de fatores genéticos e ambientais, sendo seu valor influenciado principalmente pela concentração de carboidratos (açúcares e amido) (MARTINSEN e SCHAARE, 1998). Os valores de sólidos solúveis das variedades C e D diferiram entre si e das demais, tendo a variedade $C$ apresentado o menor teor $\left(8,68^{\circ} \mathrm{Brix}\right)$. $A$ variedade $D$ revelou o maior valor para acidez titulável, quando comparada com as outras possíveis variedades. Já a variedade $B$ apresentou a menor acidez (2,55 gramas de ácido cítrico em 100 gramas de fruto), havendo diferença de 3,21 entre as variedades $D$ e B. Essa elevada acidez pode representar ponto positivo para a indústria de sucos, pois exige maior diluição do produto e, por conseguinte, maior rendimento do produto final (ANDRADE, ARAGÃO e FERREIRA, 1993). Para o ratio ou relação 'Brix/Acidez titulável, o valor encontrado para a variedade $B$ foi 2,85 vezes maior que o verificado para a variedade $C$, que não diferiu da variedade D (Tabela 1). De acordo com Chitarra (2000), o ratio relaciona a qualidade do fruto em termos de maturidade e sabor, aumentando conforme a maturação e adquirindo sabor mais doce. Caso as quatro variedades estivessem no mesmo estádio de maturação, a variedade B teria o sabor mais doce.

$\mathrm{O}$ teor de ácido ascórbico mostrou-se superior na variedade $\mathrm{D}$, seguido pelas variedades A, B e D. Todos os resultados diferiram estatisticamente entre si, abrangendo de 47,38 a 13,05 para as variedades $D$ e $C$, respectivamente. Considera-se razoável o teor encontrado para a variedade D (47,38 mg $\left.100 \mathrm{~g}^{-1}\right)$, visto que a legislação brasileira (BRASIL, 1998) recomenda a ingestão de $60 \mathrm{mg}$ de vitamina $\mathrm{C}$ por dia para adultos. O peso médio da variedade $\mathrm{D}(34,32 \mathrm{~g})$ indica $\mathrm{O}$ fornecimento de $27 \%$ de ácido ascórbico por fruto, o que o torna fonte alternativa natural desse ácido para a população da região. Os frutos apresentaram atividade de água (aw) na mesma ordem de grandeza, em torno de 0,97 , sem diferença estatística entre si (Tabela 1 ).

$\mathrm{Na}$ Tabela 2 encontram-se os resultados para determinação de cor das possíveis variedades de cambuci com referência aos parâmetros luminosidade, croma e ${ }^{\circ} h u e$. 
Em relação à cor, ocorreu padronização dos resultados quanto aos parâmetros luminosidade, croma e ohue. As variedades $B$ e $C$ não apresentaram diferença significativa, e as variedades $A$ e $D$ diferiram entre si e das demais variedades.

\section{TABELA 2 - DETERMINAÇÃO DA COR DAS QUATRO PROVÁVEIS VARIEDADES DE CAMBUCI}

\begin{tabular}{cccc}
\hline \multirow{2}{*}{ Variedades } & \multicolumn{3}{c}{ Cambuci } \\
\cline { 2 - 4 } & Luminosidade & Croma & ${ }^{\circ}$ Hue \\
\hline A & $47,54^{\mathrm{a}} \pm 0,71$ & $13,67^{\mathrm{a}} \pm 0,71$ & $99,88^{\mathrm{a}} \pm 0,53$ \\
B & $50,70^{\mathrm{b}} \pm 0,49$ & $17,12^{\mathrm{b}} \pm 1,18$ & $105,67^{\mathrm{b}} \pm 1,00$ \\
C & $50,59^{\mathrm{b}} \pm 0,20$ & $18,06^{\mathrm{b}} \pm 0,79$ & $104,66^{\mathrm{b}} \pm 1,06$ \\
D & $49,32^{\mathrm{c}} \pm 0,93$ & $22,08^{\mathrm{c}} \pm 0,36$ & $102,69^{\mathrm{c}} \pm 0,81$ \\
\hline
\end{tabular}

Diferentes letras minúsculas na mesma coluna indicam diferença estatística $(p<0,05)$ entre as possíveis cultivares de cambuci.

A luminosidade determinada instrumentalmente varia na escala de cor do 0 a 100 . Por meio dessa escala pode-se avaliar o quanto a amostra é mais escura (valores mais baixos) ou mais clara (valores mais altos) (KONICA MINOLTA, 1998). De acordo com a Tabela 2 houve pouca diferença de luminosidade entre as variedades A e B, com valores de 47,54 e 50,70, respectivamente, que representam boa capacidade de refletir a luz.

O croma, ou saturação, constitui o componente radial de uma representação polar das coordenadas CIELAB (MACHADO, 2010). A variedade D apresentou o maior valor de croma $(22,08)$, diferindo das demais (Tabela 2). Esse parâmetro mede o grau de afastamento do cinza e sua escala varia do zero para a cor acinzentada a 60 para a cor pura (KONICA MINOLTA, 1998).

O hue, ou matiz, é o componente angular de uma representação polar das coordenadas CIELAB (MACHADO, 2010), cujo valor possibilita situar um ponto num grupo de cor, permitindo visualizar a mudança na cor dos frutos (AZZOLINI, JACOMINO e BRON, 2004). Essa variação de cor depende do ângulo. Para o verde varia entre 70 a $165^{\circ}$ hue, conforme simulações no sítio de internet Colblindor efetuadas por Moraes-de-Souza (2011). Verificou-se que a variedade A apresentou o menor valor $\left(99,88^{\circ}\right.$ hue), tendendo ao amarelo (Tabela 2$)$.

$\mathrm{Na}$ Tabela 3 são apresentados os resultados da caracterização da geleia de cambuci, processada a partir de uma única possível variedade denominada A. Foram elaboradas duas diferentes formulações com variação na quantidade de polpa do fruto e sacarose nas proporções respectivas de $50 \% 50 \%$ e $40 \% 60 \%$.

A formação do gel sofre influência da concentração de açúcar, pectina, $\mathrm{pH}$ e acidez do produto. De acordo com Silva (2000), a geleificação proporciona melhor consistência ao produto em $\mathrm{pH}$ em torno de 3,0. Para valores mais baixos, a resistência do gel diminui enquanto que em valores maiores que 3,5 não há a formação gel. Apesar da amostra com $50 \%$ de açúcar ter mostrado diferença nas análises de $\mathrm{pH}$ e acidez em relação à amostra com $60 \%$, ambas apresentaram excelente geleificação (valores ideais para formação de gel).

O teor de proteína bruta das geleias apresentou valores baixos e as duas formulações não revelaram diferença significativa, tendo em vista a variação de apenas $10 \%$ na concentração de açúcar entre ambas (Tabela 3).

Com relação à análise de cor da geleia, as duas formulações evidenciaram diferença significativa quanto aos parâmetros luminosidade e ohue. A formulação com $60 \%$ de açúcar apresentou menor resultado quando comparado com a amostra com $50 \%$, caracterizando produto de coloração mais escura, com maior capacidade de absorção de luz e menor reflexão de luz ao ambiente. Para o croma, devido à grande variação dos resultados, as amostras não diferiram estatisticamente, revelando valores de $25,06 \pm 2,65$ e 19,82 $\pm 4,67$ para as geleias com $50 \%$ e $60 \%$ de açúcar, respectivamente (Tabela 3). 
TABELA 3 - CARACTERIZAÇÃO DA GELEIA DE CAMBUCI VARIEDADE A

\begin{tabular}{ccc}
\hline \multirow{2}{*}{ Análises } & \multicolumn{2}{c}{ Geleia de Cambuci } \\
\cline { 2 - 3 } & $\mathbf{5 0 \%}$ & $\mathbf{6 0 \%}$ \\
\hline $\mathrm{pH}$ & $3,02^{\mathrm{a}} \pm 0,01$ & $3,10^{\mathrm{b}} \pm 0$ \\
\hline Acidez $\left(\mathrm{g}\right.$ ácido cítrico $\left.100 \mathrm{~g}^{-1}\right)$ & $2,69^{\mathrm{a}} \pm 0,01$ & $1,87^{\mathrm{b}} \pm 0,02$ \\
\hline Proteína bruta $(\%)$ & $0,22^{\mathrm{a}} \pm 0,31$ & $0,22^{\mathrm{a}} \pm 0,31$ \\
\hline Luminosidade & $43,08^{\mathrm{a}} \pm 1,35$ & $36,20^{\mathrm{b}} \pm 0,95$ \\
\hline Croma & $25,06^{\mathrm{a}} \pm 2,65$ & $19,82^{\mathrm{a}} \pm 4,67$ \\
\hline oHue & $76,38^{\mathrm{a}} \pm 0,78$ & $66,28^{\mathrm{b}} \pm 0,87$ \\
\hline
\end{tabular}

Diferentes letras minúsculas na mesma coluna indicam diferença estatística $(p<0,05)$ entre as geleias de cambuci.

Na Tabela 4 são apresentadas as médias dos valores obtidos no teste de aceitação das geleias, realizado por 52 julgadores.

TABELA 4 - TESTE DE ACEITAÇÃO DA GELEIA DE CAMBUCI VARIEDADE A

\begin{tabular}{ccc}
\hline \multirow{2}{*}{ Análises } & \multicolumn{2}{c}{ Geleia de Cambuci } \\
\cline { 2 - 3 } & $\mathbf{5 0 \%}$ & $\mathbf{6 0 \%}$ \\
\hline Aparência & $4,5^{\mathrm{a}}$ & $5,62^{\mathrm{b}}$ \\
Cor & $4,23^{\mathrm{a}}$ & $5,77^{\mathrm{b}}$ \\
Aroma & $4,54^{\mathrm{a}}$ & $5,00^{\mathrm{a}}$ \\
Sabor & $4,10^{\mathrm{a}}$ & $5,04^{\mathrm{b}}$ \\
Textura & $4,44^{\mathrm{a}}$ & $5,25^{\mathrm{b}}$ \\
\hline
\end{tabular}

Diferentes letras minúsculas na mesma linha indicam diferença estatística $(p<0,05)$.

Na Figura 1 está representado o total de notas atribuído pelos julgadores no teste de preferência para as duas formulações de geleia de cambuci, em porcentagem.

A amostra com $60 \%$ de açúcar alcançou maior aceitabilidade em relação à amostra com $50 \%$ em todos os atributos analisados, com exceção do aroma cujos resultados não diferiram estatisticamente. Para os atributos aparência, cor, sabor e textura, a amostra com $50 \%$ de açúcar diferiu significativamente da amostra com $60 \%$ em relação à aceitabilidade ao nível de $5 \%$ de significância. A elevada acidez do fruto tornou-se perceptível no produto processado, visto que a geleia com maior concentração de açúcar obteve melhor aceitação pelos julgadores e apresentou diferença sensorial significativa frente à geleia com menor concentração de açúcar. Para o atributo aroma não houve influência da variação da concentração do açúcar, pois ambas as amostras não apresentaram diferença perceptível pelos julgadores. De forma global para o teste sensorial, a amostra com $50 \%$ de açúcar obteve índice médio de aceitação de $62,31 \%$ e a amostra com $60 \%$ de açúcar de 76,23\% quanto aos atributos analisados (Tabela 4). Segundo Dutcoksky (1996), o índice mínimo preconizado de aceitabilidade é de $70 \%$ e somente a amostra com maior teor de açúcar atingiu esse índice.

Os totais de notas obtidos pelas amostras de geleia com $50 \%$ e $60 \%$ de sacarose foram 12 e 40, respectivamente, indicando percentual de preferência de $23 \%$ para a geleia com $50 \%$ de sacarose e $77 \%$ para a geleia com $60 \%$ de sacarose. A amostra com $60 \%$ de sacarose foi a preferida e apresentou diferença significativa da amostra com $50 \%$ ao nível de $5 \%$ de significância. O aumento da concentração de açúcar proporcionou abrandamento na acidez do produto e melhoria nas características sensoriais da geleia. 


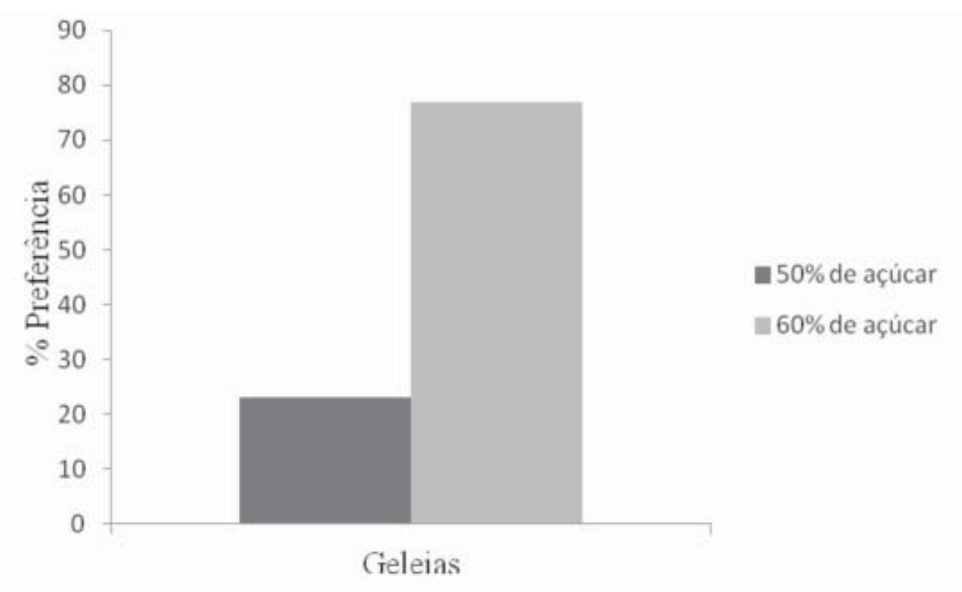

FIGURA 1 - TESTE DE PREFERÊNCIA DAS GELEIAS DE CAMBUCI

\section{CONCLUSÃO}

Considerando os dados obtidos conclui-se que somente a atividade de água não apresentou diferença significativa para as possíveis variedades de cambuci. A acidez e o ratio da variedade $B$ chamam a atenção e indicam fruto mais propício ao consumo in natura. Em todas as outras análises verificou-se diferença estatística de pelo menos uma variedade. Tais dados devem ser vistos com cautela devido influência das condições edafoclimáticas, o que aponta para estudos específicos para afirmar a existência de diferentes variedades de cambuci.

Das geleias analisadas, a amostra com $60 \%$ de açúcar apresentou resultados menores para $\mathrm{pH}$, acidez, luminosidade e 'hue, o que contribuiu para os resultados de aceitação no teste de preferência em todos os atributos analisados (aparência, cor, sabor e textura), exceto com relação ao aroma em que ambas as amostras (50\% e $60 \%$ de açúcar) não diferiram. De forma geral, a maior concentração de açúcar influenciou positivamente as notas dos julgadores, tendo a geleia com $60 \%$ de açúcar obtido maior aceitação.

\section{ABSTRACT \\ CHARACTERIZATION STUDY OF CAMBUCI FRUIT [Campomanesia phaea (O. Berg.) Landrum] AND ITS APPLICATION IN JELLY PROCESSING}

The objective of this paper was to study possible differences of varieties of cambuci fruit reported by producers by means of fruit characterization and elaboration of jelly. The fruits were divided in four possible varieties groups, named as $\mathrm{A}, \mathrm{B}, \mathrm{C}$ and $\mathrm{D}$, and submitted to analysis regarding weight, ash, moisture, $\mathrm{pH}$, soluble solids, acidity, ratio, ascorbic acid and water activity. To the preparation of the jelly was chosen the variety A with two formulations, $50 \% 50 \%$ and $40 \% 60 \%$, fruit and sugar respectively. Concerning the possible varieties of cambuci only water activity was not significantly different. The acidity and ratio of B variety are noteworthy, because it did differ from the others presenting a more satisfactory result for in natura consumption. All other tests presented statistic alteration of at least one variety, but since these data can be influenced by edaphoclimatic conditions they must be carefully observed. Lower results for $\mathrm{pH}$, acidity, luminosity and ohue, were observed for the $60 \%$ sugar jelly sample, which contributed for its better results in the preference test for the attributes appearance, color, flavor and texture. The evaluation of attribute aroma of both samples (50\% and $60 \%)$ did not differ.

KEY-WORDS: Campomanesia phaea; MYRTACEAE; JELLY; SENSORY ANALYSIS.

\section{REFERÊNCIAS}

1 ANDRADE, J. de S.; ARAGÃO, C.G.; FERREIRA, S.A. do N. Caracterização física e química dos frutos de Araçá-Pêra (Psidiumacutangulum D. C.). Acta Amazônica, Manaus, v.23, n. 2-3, p. 213-217, 1993.

2 AOAC. Association of Official Analitical Chemistry. Official methods of analysis of the AOAC International. $18^{\text {th }}$ ed. Washington, 2005. 
3 AZZOLINI, M.; JACOMINO, A.P.; BRON, I.U. Índices para avaliar qualidade pós-colheita de goiabas em diferentes estádios de maturação. Pesquisa Agropecuária Brasileira, Brasília, v.39, n.2, p.139-145, 2004.

4 BELTRÃO FILHO, E. M; SILVA, M. do L.P. da. Processamento de doce em massa de banana (Musa sp) - cultivar nanicão. In: JORNADA NACIONAL DA AGROINDÚSTRIA. 2006, Bananeiras. Anais... Bananeiras: UFPB, 2006.6 p.

5 BRASIL. Ministério da Saúde. Portaria n. 33 de 13 de janeiro de 1998. Fornece os princípios gerais para o estabelecimento de níveis máximos de ingestão diária em alimentos. Diário Oficial [da] República Federativa do Brasil, Brasília, DF, 16 jan. 1998 Seção I-E, p. 5.

6 CHITARRA, M.I.F. Tecnologia e qualidade de pós-colheita de frutos e hortaliças. Lavras: UFLA/FAEPE, 2000. 68 p.

7 DUTCOKSKY, S. D. Análise sensorial de alimentos. Curitiba: Champagnat, 1996. 123 p.

8 FRANCO, G. Tabela de composição de alimentos. 9. ed. São Paulo: Atheneu, 1999. 303 p.

9 KAWASAKI, M.L.; LANDRUM, L.R. A rare and potentially economic fruit of Brazil: cambuci, Campomanesia phaea (Myrtaceae). Economic Botany, v. 51, n. 4, p. 403-407, 1997.

10 KONICAMINOLTA. Comunicação precisa da cor: controle de qualidade da percepçãoà instrumentação. Tokyo, 1998. 59 p.

11 LEGRAND, C.D.; KLEIN, R.M. Mirtáceas: Campomanesia. In: REIZ, P.R. Flora ilustrada catarinense. Itajaí: Herbário Barbosa Rodrigues, 1977. p. 573-623.

12 LIMA, A.C.; GARCIA, N.H.P.; LIMA, J.R. Obtenção e caracterização dos principais produtos do caju. Boletim do CEPPA, Curitiba, v. 22, n. 1, p. 132-44, 2004.

13 MACHADO, A.F. Identificação e determinação da atividade antioxidante de carotenóides e antocianinas de frutas. 2010. 224 f. Tese (Doutorado em Ciência de Alimentos) - Universidade Estadual de Campinas, Campinas, 2010.

14 MALUF, A.M.; EREIO, W.A.P. Secagem e armazenamento de sementes de Cambuci. Pesquisa Agropecuária Brasileira, v.40, n. 7, p. 707-714, 2005.

15 MARTINSEN, P.; SCHAARE, P. Measuring soluble solids distribution in kiwifruit using near-infrared imaging spectroscopy. Postharvest Biology and Technology, v.14, n. 3, p. 271-281, 1998.

16 MATHIAS, J.; ANDRADE, G.A. de. Cambuci: nativa da mata atlântica, a árvore frutífera é também uma planta ornamental, mas está sob risco de extinção. Disponível em: <http://revistagloborural.globo.com/GloboRural/0,6993,EEC 16494924529,00 .html>. Acesso em: 14/04/2011.

17 MEDINA, J. C. Cultura da banana: cultura, matéria-prima, processamento e aspectos econômicos. Campinas: ITAL, 1985. $131 \mathrm{p}$.

18 MEILGAARD, M.; CIVILLE, V.; CARR, B.T. Sensory evaluation techniques. $2^{\text {nd }}$ ed. Boca Raton: CRC Press, 1991.354 p.

19 MORAES-DE-SOUZA, R. A. Qualidade de polpa de camu-camu [Myrciaria dubia (H.B.K.) McVaugh], submetida aos processos de congelamento, pasteurização, alta pressão hidrostática e liofilização e armazenada por quatro meses. 2011. 115 f. Tese (Doutorado em Química na Agricultura e no Ambiente) - Centro de Energia Nuclear na Agricultura, Piracicaba, 2011.

20 PORTAL ACTION. Teste de Tukey (TSD - Tukey Significant Difference). Disponível em: <http://www.portalaction.com. br/content/31-teste-de-tukey>. Acesso em: 10/03/2011.

21 SILVA, J.A. Tópicos da tecnologia dos alimentos. São Paulo: Livraria Varela, 2000. 227 p.

22 SLOW FOOD BRASIL. Cambuci. [online] Disponível em: <http://www. slowfoodbrasil. com/content/ view/328/59/>. Accesso em: 14 abril 2011.

23 SOUZA, M.L.; MENEZES, H.C. Processamento de amêndoa e torta de castanha-do-Brasil e farinha de mandioca: parâmetros de qualidade. Ciência e Tecnologia de Alimentos, v. 24, n. 1, p. 120-8, jan.-mar. 2004.

24 STROHECKER, R.; HENNING, H.M. Analisis de vitaminas: métodos comprobados. Madrid: Paz Montalvo, 1967. 428 p.

25 VALLILO, M.I.; GARBELOTTI, M.L.; OLIVEIRA. E. de; LAMARDO, L.C.A. Características físicas e químicas dos frutos do cambucizeiro (Campomanesia phaea). Revista Brasileira de Fruticultura, v. 27, n. 2, p. 241-244, 2005.

\section{AGRADECIMENTOS}

Zé Ferro, produtor da região de Mogi das Cruzes no interior do estado de São Paulo. 